\title{
L'ingegneria sanitaria ambientale a supporto dell'agricoltura urbana per lo sviluppo di un'economia circolare
}

\author{
Francesco Bovenzi ${ }^{1}$ \\ ${ }^{1}$ Affiliation not available
}

\begin{abstract}
Secondo uno studio della FAO entro il 2050 ci sarà un forte aumento della popolazione mondiale, il che porterà a conseguenze gravi quali un aumento sostanziale della domanda di cibo, la riduzione della risorsa idrica e un eccessivo consumo del suolo. In questi quadro ricopre un ruolo di interesse l'agricoltura urbana, essa consiste nel coltivare cibo all'interno di contesti urbani. Si ricercherà sempre più spesso un legame tra agricoltura ed economia circolare, essa consiste in un'economia pensata per potersi rigenerare da sola. L'unione tra economia circolare e agricoltura urbana porta i BIA che consistono in edifici che ospitano al proprio interno delle serre. Si pone quindi come necessaria la transizione dal modello lineare ad un modello circolare, che a considerazione di tutte le fasi sappia limitare l'apporto di materia ed energia in ingresso e di minimizzare scarti e perdite.
\end{abstract}

\section{Agricoltura urbana}

Si sente parlare sempre più spesso di agricoltura urbana, essa consiste nel coltivare il cibo all'interno di contesti urbani o periurbani come città o villaggi.

Stiamo assistendo ad un forte sviluppo dell' agricoltura urbana all'interno delle città. Questo poiché vi è una continua ricerca per trovare un legame tra sostenibilità e vivibilità. Possiamo stabilire che 
essa ha tre funzioni principali:

- Ambientale, in quanto vi è una migliore gestione delle risorse naturali;

- Miglioramento della sanità pubblica, poiché vi è un aumento della frutta e della verdura di fresco consumo,

- Economico, in quanto vi è una riduzione dei costi di trasporto.

L'agricoltura urbana rappresenta un'opportunità concreta ed accessibile, per i residenti delle città di esse coinvolti in questioni di provenienza e sicurezza alimentare e di riconnettersi con un sistema alimentare che molti ritengono fuori dalla loro portata ${ }^{1}$.

Esiste una vasta varietà di approcci all'agricoltura urbana che cambiano in base alle specifiche condizioni ambientali, sociali ed economiche. Come approcci conosciamo:

- L'agricoltura a terra, essa può risolvere il problema dei lotti di terreno troppo piccoli per essere edificati. Infatti va ad occupare quelle aree abbandonate trasformandole in risorse per la comunità, ciò può portare alle città numerosi vantaggi sotto il profilo dei costi dei prodotti per il consumatore oc addirittura vantaggiosi per le proprietà che circondano le aree verdi in quanto vi sarà un aumento del valore del lotto ${ }^{2}$

- Rooftop agricolture, i vincoli territoriali delle aree urbane hanno portato allo sviluppo di siti alternativi per la produzione alimentare, in particolare i tetti. il vantaggio principale delle colture sui tetti consiste in un controllo maggiore sui potenziali contaminanti, consentendo quindi una gestione abbastanza efficace sulla composizione dei substrati e sui nutrienti;

- Controlled Evioriment Agricolture (CEA), è un approccio all'agricoltura urbana che ha suscitato un crescente interesse, in particolare modo nelle regioni in cui gli inverni freddi limitano la crescita all'aperto. Nelle aree urbane spesso si svolge sui tetti in quanto i costi dei terreni sono molto elevati(fig.1).

Infine sistemi all'avanguardia sanno sempre più prendendo piede, quali i sistemi acquaponici: al- 
cuni studi condotti ne evidenziano la funzionalità del processo e la sua applicabilità. ${ }^{34}$

\section{Economia Circolare}

L'obiettivo della ricerca è stato quello di trovare un legame tra economia circolare e agricoltura urbana. L'economia circolare è definita come un modello economico pensato per potersi rigenerare da solo, garantendo così la sua eco-sostenibilità ${ }^{5}$.

Tale concetto risponde al desideri di crescita sostenibile, nel quadro della pressione crescente a cui produzione e consumi sottopongono le risorse mondiali e l'ambiente ${ }^{6}$. Il nuovo modello economico implica condivisione, prestito riutilizzo, riparazione, ricondizionamento e ricircolo dei materiali e prodotti esistenti. in questo modo si estende il ciclo di vita dei prodotti contribuendo a ridurre al minimo i rifiuti.

Adottare un approccio circolare significa rivedere tutte le fasi della progettazione e prestare attenzione all'intera filiera coinvolta nel ciclo produttivo ${ }^{7}$. Questa attenzione passa per 5 criteri fondamentali:

- Eco-progettazione, progettare i prodotti pensando fin da subito al loro impiego a fine vita;

- Modularità e versatilità, dare priorità a questa affinché il prodotto si possa adattare al cambiamento delle condizioni esterne;

- Energie rinnovabili, affidarsi ad energie prodotte da fonti rinnovabili favorendo così il rapido abbandono del modello energetico fondato dalle fonti fossili;

- Approccio eco-sistemico, pensare in maniera olistica facendo attenzione all'intero sistema e considerando le relazioni causa-effetto;

- Recupero dei materiali, favorire la sostituzione delle materie prime vergini con materie già utilizzate provenienti da filiere di recupero che ne conservano la qualità. 
Questo concetto si comprende al meglio osservando i sistemi viventi naturali, che funzionano in modo ottimale poiché ognuno dei loro elementi si inserisce bene nel complesso. I prodotti sono progettati appositamente per essere inseriti nei cicli di vita riducendo così al minimo i rifiuti residui.

\section{Effetti ambientali dei sistemi idroponici}

L'intensificazione dei sistemi di produzione alimentare per soddisfare la domanda di cibo sta causando importanti impatti ambientali, come l'aumento delle emissioni dei gas serra o l'esaurimento dell' acqua sia a livello globale che locale.$^{8}$

Un approccio di economia circolare applicato all'agricoltura urbana potrebbe contribuire a mitigare questi impatti. La chiusura del ciclo dei nutrienti nei sistemi fuori suolo mantiene l'utilità e il valore delle scarse risorse e produce un effetto rigenerativo sull' ambiente. Sistemi idroponici possono far ricircolare le sostanze pene di nutrienti grazie ad un sistema di irrigazione e raccolta delle acque a circuito chiuso.

Nel campus dell'Università Autonoma de Barcelona a $15 \mathrm{~km}$ dalla città spagnola è stata condotta un'analisi su due sistemi idroponici, uno lineare e uno circolare che permette il ricircolo.

Il sistema lineare consiste in un sistema di irrigazione idroponico, in cui i sacchetti di substrato sono posizionati su vassoi di polistirene, non viene aggiunto nessun trattamento per il percolato. Nel caso del sistema circolare invece i sacchetti vengono posizionati su vassoi di alluminio inclinati, per permettere al percolato di cadere dallo stesso lato. Questo viene raccolto e inviato in un serbatoio di 300L, successivamente viene filtrato tramite filtro a sabbia e abbattuta la carica batterica con una lampada UVL. Il tutto poi viene immagazzinato in un serbatoio di $30 \mathrm{~L}$ ed infine viene prelevata l'acqua ed utilizzata per l'irrigazione. Il funzionamento è schematizzato nella figura 3 .

In seguito vengono analizzate le produzioni massime, il consumo d'acqua ed il consumo dei nutrienti. Dalle analisi è emerso che le produzioni massime risultano $0,26 *$ pianta^ 1 nel caso del 
sistema lineare e 0,28* pianta^-1 nel caso del sistema circolare. Entrambe le produzioni risultano ottime secondo i dati forniti dalla letteratura per la coltivazione dei fagioli nelle serre sella Spagna durante i mesi autunnali. L'acqua utilizzata per il sistema lineare risulta pari a 38,9L*piantâ^-1 e 36,4L*pianta^-1 nel caso del sistema circolare. il risparmio d'acqua del sistema circolare è quasi del $40 \%$ ed è uno dei risultati più importanti di tale studio. Per quanto riguarda i nutrienti si è riscontrato un risparmio notevole per tutti i nutrienti, pari al $45 \%$ per $\mathrm{N}, 35 \%$ per $\mathrm{P}, 41 \%$ per $\mathrm{K}$ $53 \%$ per $\mathrm{Ca}, 51 \%$ per $\mathrm{Mg}$ e $54 \%$ per S. Tali risparmi sono degni di particolare attenzione considerando che alcuni di questi nutrienti sono strettamente legati a risorse non rinnovabili. Ad esempio il fosforo $(\mathrm{P})$ che proviene principalmente da rocce fosfate non rinnovabili.

\section{Building-Integrated Agricolture}

Esso è un nuovo approccio alla produzione alimentare che si basa sull'idea di localizzare sistemi di coltivazione altamente tecnologici su e negli edifici, utilizzando fonti di energia locali e rinnovabili. Le installazioni in genere includono funzionalità come il ricircolo dell'acqua, lo sfruttamento del calore residuo, sistemi di capostazione dell'acqua piovana e il raffreddamento per evaporazione. I componenti del sistema variano in base alle dimensioni dell'edificio, alla capacità strutturale, al clima locale e alla disponibilità della luce.

I BIA sono sistemi ecologicamente sostenibili per la produzione di cibo, riducono l'impronta ambientale, riducono i costi di trasporto, migliorano la sicurezza alimentare, raffreddano gli edifici combattendo il riscaldamento globale.

La gestione della risorsa idrica è una caratteristica importante di questi sistemi che impiegano la tecnologia idroponica a ricircolo, la raccolta delle acque piovane e l'utilizzo delle acque grigie dell'edificio ospitante. L'agricoltura idroponica è la forma più efficiente dal punto di vista idrico, consuma fino a dieci volte meno acqua rispetto ad un'agricoltura convenzionale, eliminando 
contemporaneamente l'utilizzo dei pesticidi chimici.

la qualità e la resa delle colture sono in gran parte in funzione del controllo del clima all'interno della serra. il mantenimento di livelli costanti di temperatura e umidità in una serra consente la produzione di alimenti per tutto l'anno, ma contemporaneamente rappresenta una sfida dal punto di vista energetico. Tali esigenze però possono essere soddisfatte dal fotovoltaico, esso è particolarmente adatto poiché il picco della domanda elettrica coincide con il picco della fornitura ad esempio in un caldo pomeriggio dice c'è bisogno del raffreddamento. Il carico elettrico può essere ridotto utilizzando un sistema di ventilazione naturale o pompe e ventilatori ad alta efficienza.

Bisogna considerare poi un'ulteriore aspetto legato controllo degli odori e della carica patogena che spesso sono problematiche comuni nelle pratiche agricole convenzionali e non ${ }^{910}$.

\section{Conclusioni}

Trovare modi per migliorare le prestazioni dei sistemi agricoli nel quadro dell'approvvigionamento alimentare urbano è cruciale per ottimizzare il futuro delle grandi città che seguono i principi dell'economia circolare. Per lo studio dei sistemi idroponici sono state valutate alcune conclusioni.

In primo luogo i sistemi di irrigazione a ricircolo aiutano a ridurre le quantità d'acqua utilizzate per l'irrigazione, alleviando coì gli effetti della scarsità della risorsa idrica.

La gestione dei nutrienti è ottimizzata dai sistemi circolari, inoltre questi non vengono scaricati nell'ambiente evitando così l'eutrofizzazione, cosa che nei sistemi lineari avviene. Sono stati riscontrati ovviamente risparmi anche sulla quantità di fertilizzanti utilizzati.

Per valutare le possibilità di miglioramento in questi sistemi agricoli urbani su larga scala e con un ampia varietà di colture occorre studiare più strategie di distribuzione della risorsa idrica. Inoltre, in altre ricerche occorre un confronto dei costi del ciclo di vita tra sistemi lineari e circolari, in 
quanto il produttore darà sempre la priorità al fattore costo.

\section{References}

1.Naddeo, V., Belgiorno, V., Zarra, T. \& Scannapieco, D. Dynamic and embedded evaluation procedure for strategic environmental assessment. Land Use Policy 31, 605-612 (2013).

2.I.Voicu \& Been, V. The Effect of Community Gardens on Neighboring Property Values. Real Estate Economics 36, 241-283 (2008).

3.Regno, M. D. Processi naturali per il recupero e il riutilizzo delle acque nei sistemi acquaponici. doi:10.22541/au.158525193.30158307.

4.Muro, G. D. Verso una agricoltura urbana sostenibile: i sistemi acquaponici per il recupero e il riutilizzo delle acque . doi:10.22541/au.158525190.07859900.

5.V.Naddeo, Balakrishnan, M. \& K.H.Choo. Frontiers in Water-Energy-Nexus-Nature-Based Solutions, Advanced Technologies and Best Practices for Environmental Sustainability. (2018).

6.Nesticò, A., Elia, C. \& Naddeo, V. Sustainability of urban regeneration projects: Novel selection model based on analytic network process and zero-one goal programming. Land Use Policy 99, 104831 (2020).

7.Scannapieco, D., Naddeo, V. \& Belgiorno, V. Sustainable power plants: A support tool for the analysis of alternatives. Land Use Policy 36, 478-484 (2014).

8.Foley, A. \& Ramankutty, N. Solutions for a cultivated planet. Nature 478, 337-342 (2011).

9.T.Zarra, V.Naddeo \& V.Belgiorno. A novel tool for estimating the odour emissions of composting plants in air pollution management. Issue 4 11, 477-486 (2013).

10.V.Naddeo, T.Zarra, V.Giuliani \& V.Belgiorno. Odour Impact Assessment in Industrial Areas. 
Cet 30, (2012).

11.Naddeo, V. Development of environmental biotechnology and control of emerging biological contaminants: the grand challenge for a sustainable future. Water Environment Research 92 , 1246-1248 (2020). 


\section{Figure Captions}

Figure 1. Controlled Evioriment Agricolture (CEA)

Figure 2. Economia circolare ( Eureopean Parlament)

Figure 3. Schematizzazione dei due sistemi 
Figures

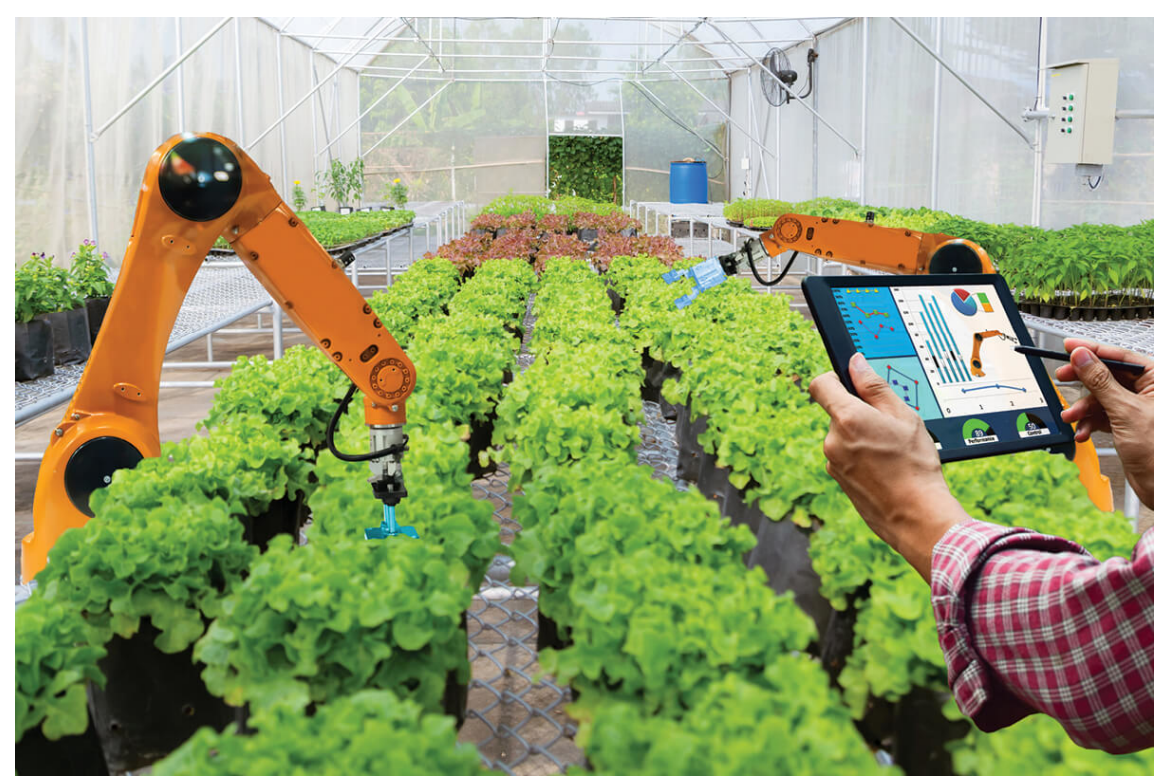

Figure 1: Controlled Evioriment Agricolture (CEA) 


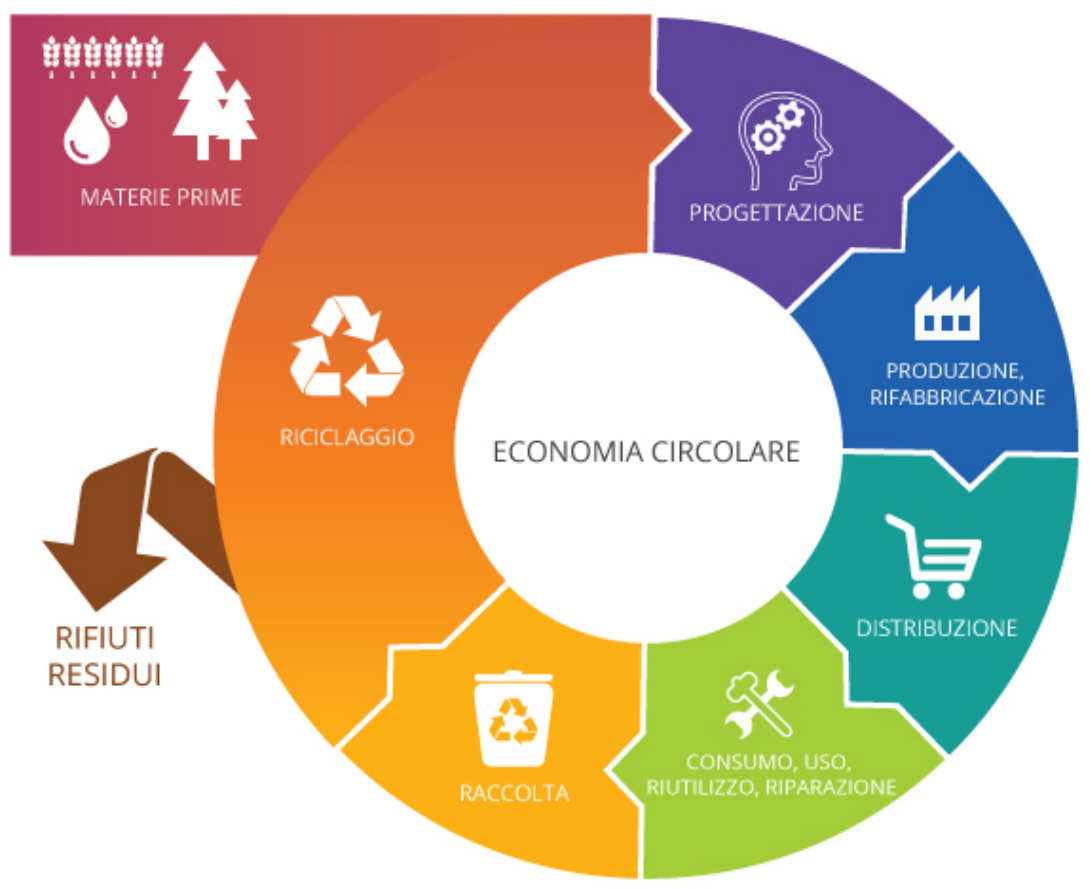

Figure 2: Economia circolare ( Eureopean Parlament) 


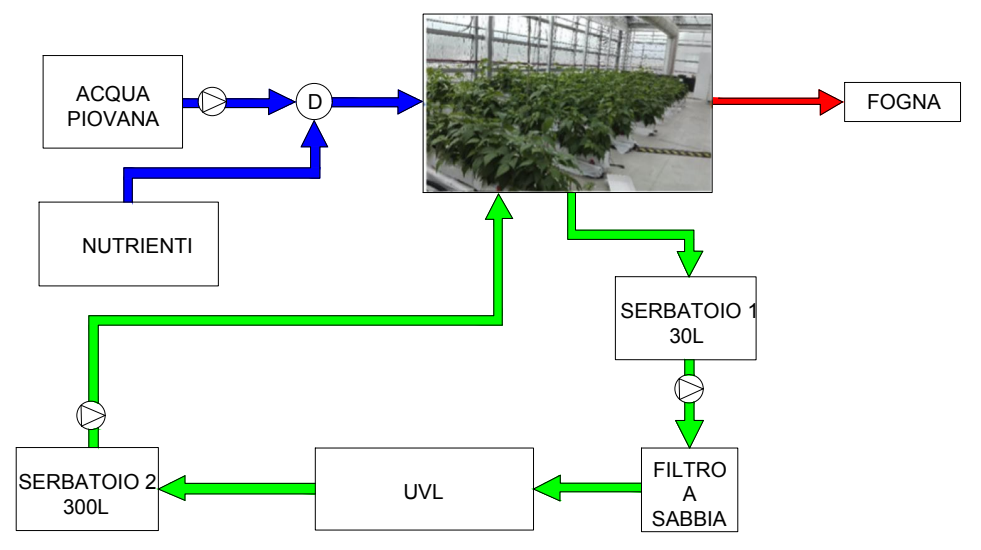

Figure 3: Schematizzazione dei due sistemi 